\title{
A Mathematical Model of Trophoblast Invasion
}

\author{
H. M. BYRNE ${ }^{a, *}$, M. A. J. CHAPLAIN ${ }^{b, \dagger}$, G. J. PETTET ${ }^{c} \ddagger$ and D. L. S. McELWAIN ${ }^{c}, \mathbb{I}$ \\ a Department of Mathematics, UMIST, PO Box 88, Manchester M60 1QD; ${ }^{b}$ Centre for Nonlinear Systems in Biology, Department of \\ Mathematics, University of Dundee, Dundee DDI $4 \mathrm{HN} ;{ }^{\circ}$ Centre in Statistical Science and Industrial Mathematics, School of \\ Mathematical Sciences, Queensland University of Technology. Brisbane, Qld 4001 Australia
}

(Received 30 April 1998; In final form 30 April 1998)

\begin{abstract}
In this paper we present a simple mathematical model to describe the initial phase of placental development during which trophoblast cells invade the uterine tissue as a continuous mass of cells. The key physical variables involved in this crucial stage of mammalian development are assumed to be the invading trophoblast cells, the uterine tissue, trophoblast-derived proteases that degrade the uterine tissue, and protease inhibitors that neutralise the action of the proteases. Numerical simulations presented here are in good qualitative agreement with experimental observations and show how changes in the system parameters influence the rate and degree of trophoblast invasion. In particular we suggest that chemotactic migration is a key feature of trophoblast invasion and that the rate at which proteases are produced is crucial to the successful implantation of the embryo. For example, both insufficient and excess production of the proteases may result in premature halting of the trophoblasts. Such behaviour may represent the pathological condition of failed trophoblast implantation and subsequent spontaneous abortion.
\end{abstract}

Keywords:

\section{INTRODUCTION}

The implantation of the mammalian embryo into maternal uterine tissue and the subsequent formation of the placenta play a crucial role in any successful pregnancy. The process of embryo implantation is characterised by a controlled sequence of events during which the trophoblast cells of the developing placenta interact with the uterine stroma in a carefully orchestrated fashion (Bischof and Campana, 1996; Burrows et al., 1996; Carlson, 1994; Endos, 1991; Harvey et al, 1995; Redman, 1997; Strickland and Richards, 1992) (see Figure 1 for a schematic diagram of this process). Initially trophoblast cells proliferate and migrate as a continuous mass bearing numerous tips that form villi. Anchoring villi are formed when these tips make contact with the maternal decidua. Trophoblast cells

\footnotetext{
*Corresponding Author: E-mail: h.byrne@umist.ac.uk

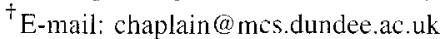

士E-mail: g.pettet@fsc.qut.edu.au

IE-mail: s.mcelwain@fsc.qut.edu.au
} 


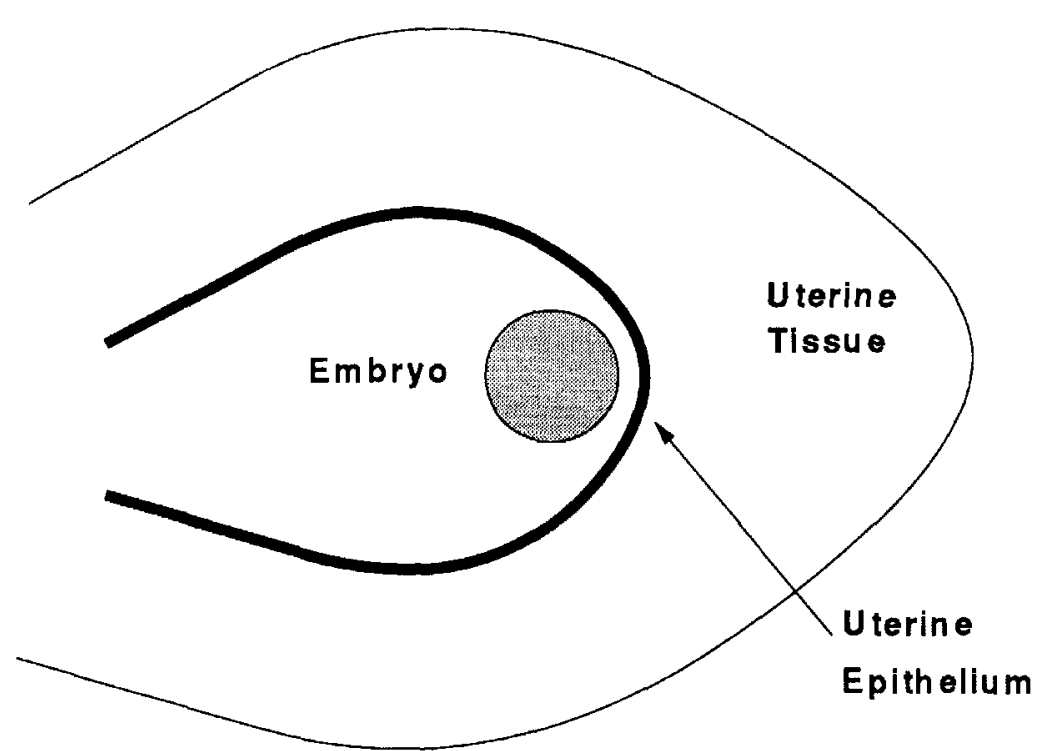

FIGURE 1 Schematic diagram (not to scalc) showing the implantation of the early cmbryo in the uterus. At this stage the embryo is in the blastocyst stage (largcly spherical) and the outer layer(s) or shell of the blastocyst is composed of trophoblast cells.

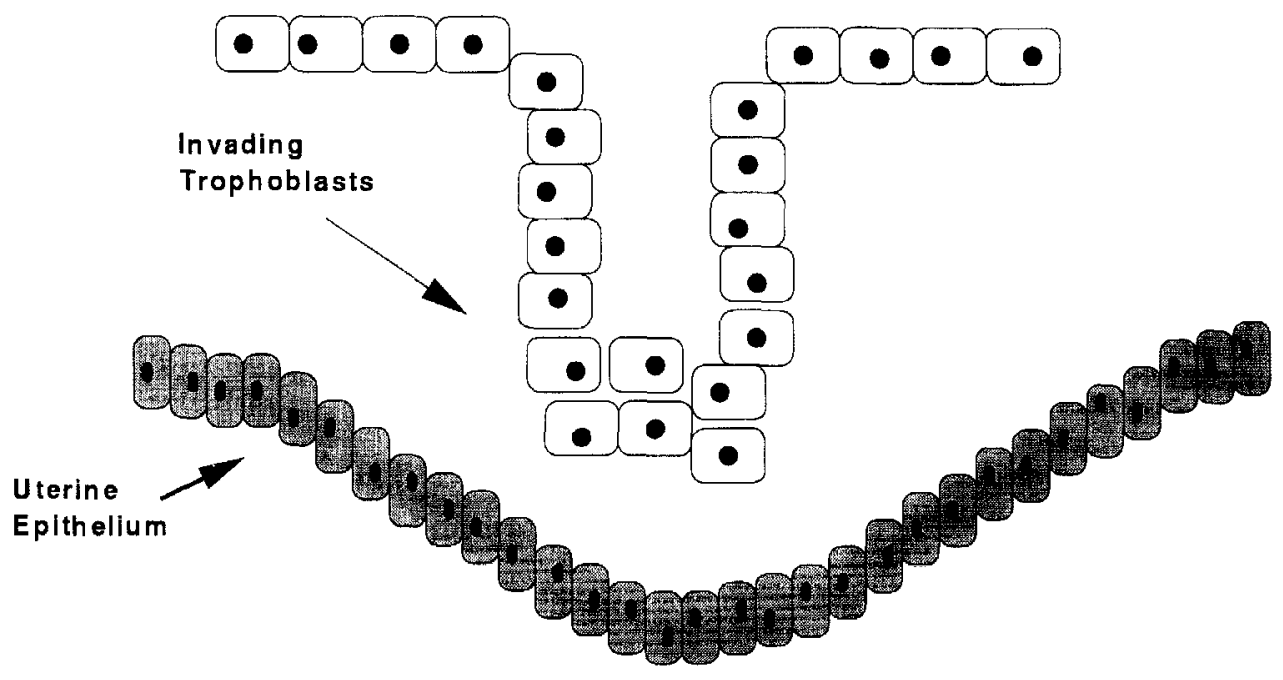

Uterine Tissue

FIGURE 2 Schemaric diagram of the trophoblast cells forming an invading tip as they migrate, proliferate and invade the uterine tissue.

in the anchoring villi continue to proliferate and form columnar aggregates. Loss of contact inhibition causes individual trophoblast cells to break away from these columns and to migrate through the maternal decidua, towards maternal blood vessels where they degrade the muscle lining of the blood vessels, thereby increasing maternal blood flow to the developing foetus (see Figure 2 for a schematic diagram of this process). Eventually the trophoblasts mature into giant cytotrophoblasts which are found 
throughout the decidua of term pregnancies. Detailed diagrams and descriptions of this stage of embryo development can be found in standard textbooks such as Carlson (1994). In this paper, we will be concerned only with the earliest stages of placental development, when the trophoblasts migrate as a continuous mass of cells.

In order to effect their invasion, trophoblast cells secrete various enzymes such as metalloproteases (MMPs), which are capable of degrading the extracellular matrix (ECM) of the uterine tissue into which the trophoblasts invade. As part of the regulatory mechanism that limits the extent of trophoblast invasion, the uterine tissue responds to its degradation by producing specific inhibitors, such as tissue inhibitor of metalloproteases (TIMPs), which neutralise the degradative enzymes (Kleiner and StetlerStevenson, 1993). Normally this carefully controlled process results in the successful implantation of the embryo (Graham and Lala, 1992) and the development of a mature placenta. Poorly regulated invasion may be important in various pathological conditions such as failed trophoblast implantation and subsequent spontaneous abortion (under-invasion) or choriocarcinoma (over-invasion).

In the following section we present a simple mathematical model that describes the invasion of trophoblast cells into the uterine tissue. The model is perhaps most applicable to placental development in pigs since porcine placentas expand into the maternal tissue as dense, almost radially-symmetric masses of trophoblast cells (cf. Figure 1). Equally our model describes experimental assays in which mice blastocysts are cultured in vitro and the spreading of trophoblast cells observed using time lapse video microscopy (Suenaga et al., 1996). The model we present here examines the interaction between four key variables: the trophoblast cells, the uterine tissue, trophoblast-derived proteases and uterine tissue-derived protease-inhibitors.

We would like to stress that our mathematical model focuses primarily on the initial stages of placental development, during which trophoblast cells proliferate and migrate as a continuous mass, forming anchoring and floating villi (cf. Figure 2).
As such it distinguishes between the ability of a mass of trophoblast cells to invade the uterine tissue and their failure to penetrate the maternal decidua. Consequently, the model does not describe the later stages of placental development, during which individual trophoblasts disaggregate from the columnar anchoring villi and remodel the underlying maternal stroma.

The model broadly reproduces the in vivo and in vitro situations described above and enables us to suggest two mechanisms which may be associated with the cause of failed trophoblast invasion. It should be possible to test the validity of these claims and, hence, the validity of our model by performing appropriate experiments in vitro.

\section{THE MATHEMATICAL MODEL}

As outlined in the previous section, placental development is a complex process involving the coordination of many mechanisms. In the mathematical model presented below we focus attention on what we believe to be the key variables involved in early trophoblast invasion, namely trophoblast cells, protease, inhibitor and uterine tissue. In its present form the model most closely describes either the development of porcine placentas or the outgrowth of trophoblast cells from murine blastocysts cultured in vitro (Suenaga et al., 1996). In this in vitro assay trophoblast spreading over a two-dimensional monolayer is caused by cell migration and proliferation.

In developing our mathematical model we make a number of simplifying assumptions and approximations. In particular, we focus on the outgrowth of the trophoblast cells that constitute the anchoring and floating villi during the early stages of placental development, before a connected vascular network has developed within the placenta. By assuming that the system is well-oxygenated we are able to ignore the effect that changes in oxygen tension exert on trophoblast migration and differentiation (Genbacev et al., 1996, 1997). In addition, rather than using separate variables to describe the action of the various chemoattractants and protease inhibitors that are 
known to be present during early placental development, we consider a single, generic chemical which acts as a chemoattractant to the trophoblast cells and also neutralises the protease. Due to the way in which it neutralises the proteases we term this chemical an inhibitor. Assuming that both protease inhibitors and chemoattractants are produced during uterine tissue degradation we anticipate that their concentration profiles within the maternal decidua will be qualitatively similar. Hence by considering a single generic chemoattractant/inhibitor it should be possible to mimic the behaviour of the invading trophoblasts: what is important is incorporating chemotactic and inhibitory effects into the model.

We denote the trophoblast density by $n$, the protease concentration by $u$, the inhibitor concentration by $v$, and the uterine tissue density by $r$. For simplicity, we restrict attention to a simple one-dimensional geometry in which the independent variables are distance from the endometrial endothelium $x$ and time $t$. Our model equations describe the evolution of $n, u, v$ and $r$ in terms of $x$ and $t$ and can be derived using the principle of conservation of mass.

\section{(a) Trophoblast Cells}

We assume that the trophoblast cells exhibit a small degree of random motion, with motility coefficient $D_{n}$, and respond chemotactically to spatial gradients in the inhibitor, with chemotactic coefficient $\chi$.

Whilst we assume that chemotaxis dominates trophoblast locomotion during early placentation (see parameter values used in numerical simulations) we believe that it is important to use a physicallymeaningful expression to describe their random motion and consequently that nonlinear diffusion is more appropriate in this context that the usual linear terms employed in equations (2) and (3). The particular form of the nonlinearity is unimportant: all that is required is that $D_{n} \propto n^{p}(p \geq 1)$ - the case $p=2$ is merely a representative example. The important features are that the speed of propagation of the trophoblast into the uterine tissue is finite and that compact support of the initial data is preserved
(Elliott and Ockendon, 1982). This means that if the initial trophoblast profile is localised in a finite region then at all subsequent times it will be confined to a finite region whose size may change over time. By contrast, if we assumed that $D_{n} \propto n^{0}$, constant, then our model would predict that the trophoblast penetrates the entire spatial domain immediately, which is not physically realistic.

Despite the fact that the uterine tissue contains many chemicals including chemoattractants, it seems unlikely that these chemicals, which are bound to the uterine tissue, direct the trophoblast cells into the uterine tissue: if the average concentration of the chemoattractants bound to the tissue is constant then there will be no spatial gradient and consequently no chemotactic response from the trophoblast cells. Recently Giannelli et al. (1997) observed that fragments of tissue matrix which had been degraded by a protease contained cleaved laminin, a chemical to which trophoblasts respond chemotactically (Giannelli et al., 1997). We suggest that a similar process happens during early placentation, with uterine tissue degradation leading to the release of various chemoattractants in a small neighbourhood of the leading front of invading trophoblasts. Since tissue degradation is a dynamic process this mechanism will give rise to a localised gradient in a chemoaltractant.

Regarding trophoblast proliferation, we assume that in the absence of any uterine tissue $(r=0$ in equation (1)) trophoblast proliferation satisfies a logistic growth law, with rate parameter $k_{1}$ and carrying capacity scaled to unity. The presence of uterine tissue leads to competition for space between the trophoblast and the uterine tissue and we model this by including a crowding term which is proportional to the product $n r$. Thus, we have

$$
\frac{\partial n}{\partial t}=D_{n} \frac{\partial}{\partial x}\left(n^{2} \frac{\partial n}{\partial x}\right)-\chi \frac{\partial}{\partial x}\left(n \frac{\partial y}{\partial x}\right)+k_{1} n(1-n-r) .
$$

(b) Protease

We assume that the protease diffuses, with constant diffusion coefficient $D_{u}$, and that it is produced by trophoblast cells at the rate $k_{2} n(1-n)$. This term localises protease production at the leading front 
of the invading trophoblasts and is consistent with experimental observations (Harvey et al., 1995). In addition we assume that the protease is neutralised by the inhibitor as a one-to-one reaction which occurs at the rate $k_{3} u v$. Thus we have

$$
\frac{\partial \mathrm{u}}{\partial \mathrm{t}}=\underset{\substack{\text { diffusion } \\ D_{u} \frac{\partial^{2} u}{\partial x^{2}}}}{\text { production }} k_{2} n(1-n)-k_{3} u v .
$$

(c) Inhibitor

As stated above, rather than considering the evolution of a range of different chemoattractants and protease inhibitors, in this model we consider a single, generic chemical which acts as a chemoattractant to the trophoblasts and which also neutralises the protease.

We assume that the inhibitor diffuses, with constant diffusion coefficient $D_{v}$, and that it is produced by that part of the uterine tissue which is being degraded by the protease. We also assume that the inhibitor neutralises the protease in a one-toone reaction. Introducing $k_{4} u r$ to denote the rate of inhibitor production, we have

$$
\frac{\partial \mathrm{v}}{\partial \mathrm{t}}=\underset{\substack{\text { diffusion } \\ D_{v} \frac{\partial^{2} v}{\partial x^{2}}}}{k_{4} u r}-\underset{\text { production }}{k_{3} u v .}
$$

(d) Uterine tissue

Neglecting random motion of the uterine tissue, we assume that it is degraded by the protease, and proliferates while competing for space with the trophoblast cells in a manner similar to that described above for trophoblast cell proliferation (see equation (1)). Thus in the absence of trophoblast cells the uterine tissue undergoes logistic growth. However the presence of trophoblast cells leads to competition for space between the two types of cells which we again model by incorporating a crowding term into the logistic growth. Using a modified logistic term with rate constant $k_{5}$ to describe uterine tissue production, and taking $k_{6} u r$ to represent the rate of tissue degradation we have

$$
\frac{\partial r}{\partial t}=\begin{gathered}
k_{5} r(1-n-r) \\
\text { replacement }
\end{gathered} \quad \begin{gathered}
k_{6} u r \\
\text { degradation }
\end{gathered}
$$

(e) Boundary and initial conditions

We consider a one-dimensional geometry, in which $x=0$ represents the uterine wall and $x=L$ a typical penetration depth for a normal placenta. We impose no-flux boundary conditions for each dependent variable at $x=0$ and $x=L$, and so we have

$$
\frac{\partial n}{\partial x}=\frac{\partial u}{\partial x}=\frac{\partial v}{\partial x}=\frac{\partial r}{\partial x}=0 \text { at } x=0, L
$$

We assume that initially a small number of trophoblast cells have penetrated a short distance $\left(x^{*} \in\right.$ $(0, L)$, say) into the uterine tissue and that the remaining space is occupied by uterine tissue alone. In particular, no protease or inhibitor is present at this stage. Specifically, we prescribe

$$
\left.n(x, 0)=\begin{array}{c}
\frac{1}{2}\left(1+\tanh \left[\left(x^{*}-x\right) / \epsilon\right]\right), \\
\text { with } u(x, 0)=0=v(x, 0), \\
\text { and } r(x, 0)=1-n(x, 0),
\end{array}\right\}
$$

where $0<\varepsilon \ll 1$ is a small parameter. The functional form for $n(x, 0)$ represents an initial trophoblast cell distribution which decreases smoothly from a scaled value of 1 at the endometrial epithelium to 0 near $x=x^{*}$, the initial depth of penetration of the trophoblast into the uterine tissue.

\section{RESULTS}

After appropriate rescaling, the model equations can be solved numerically using the method of lines and Gear's method as implemented by the NAG routine D03PCF. Given that the aim of the model is to reproduce observed events in a qualitative manner (and in the absence of experimental data), parameter values that gave realistic qualitative results were employed. However, we have assumed that the random motility coefficient for the trophoblast cells is some orders of magnitude smaller than the diffusion coefficients for the chemical species, which is not unreasonable, and estimates of the chemical diffusion coefficients/cell random motility coefficient were obtained from Bray (1992) (cf. Chaplain and Stuart, 1993). The particular valucs of the diffusion coefficients are not critical to the results or model 

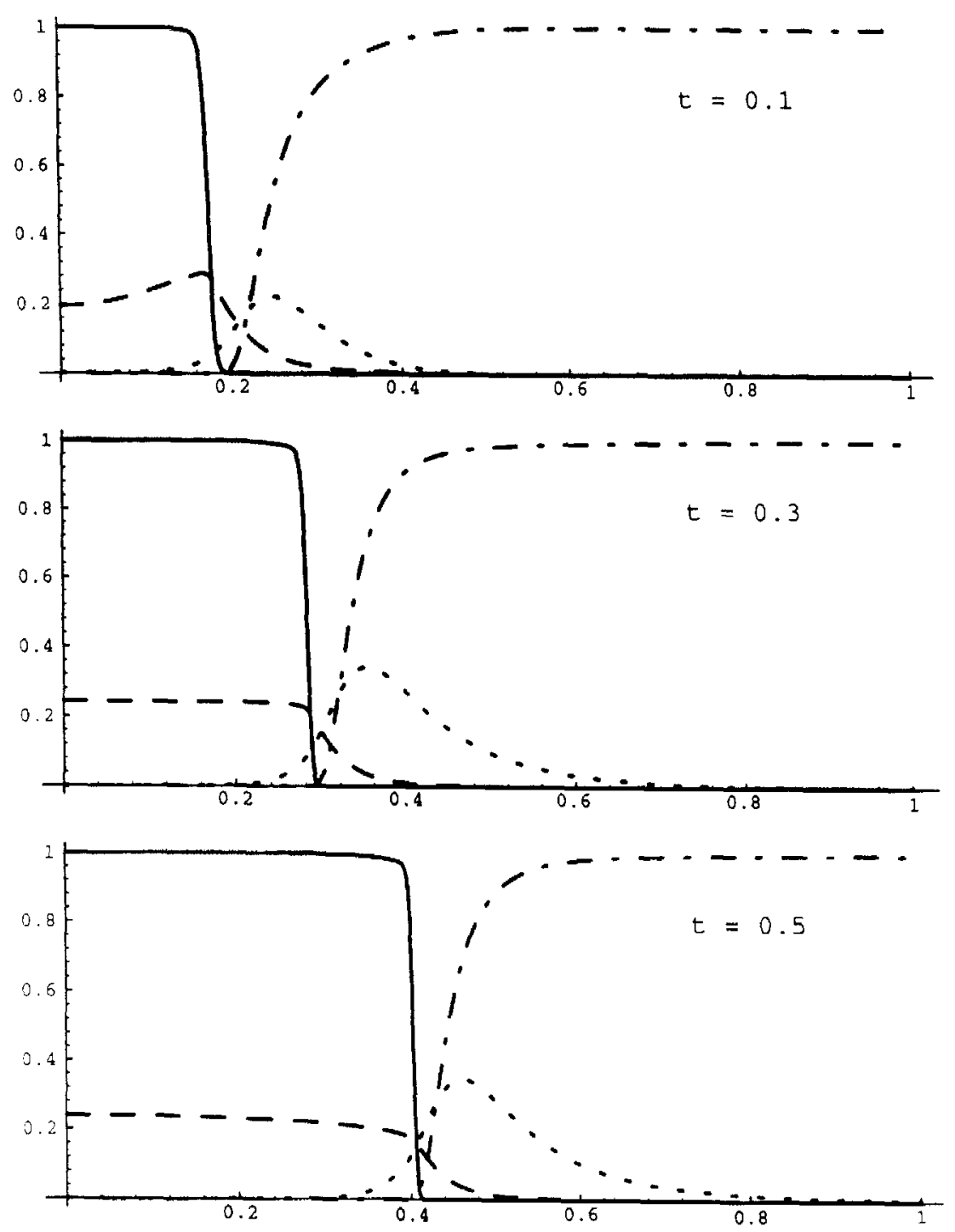

FIGURE 3 Results of a numerical simulation for which successful implantation occurs and a steady travelling wave which propagates into the uterine tissue is established. Key: trophoblasts, $n$ (solid line); protease, $u$ (dashed line): inhibitor. $v$ (dotted line); uterine tissue, $r$ (dot-dash line). Parameter values: $D_{n}=10^{-5}, D_{u}=5 \times 10^{-3}, D_{v}=10^{-3}, x=2.5 \times 10^{-3}, k_{1}=10 . k_{2}=10, k_{3}=10, k_{4}=10$, $k_{5}=10, k_{6}=100, x^{*}=0.1, \varepsilon=0.01$.

predictions, provided the orders of magnitude are maintained. What is important is the relative values of the different diffusion or random motility coefficients. The other parameter values are of a similar order of magnitude to those used in other mathematical models of invasion processes (cell migration) involving chemotaxis (cf. Chaplain and Stuart, 1993; Pettet et al., 1996a,b; Anderson and Chaplain, 1998). In the course of our simulations two types of behaviour were observed. Either the invading front of trophoblasts evolved to a travelling wave-like profile (see Figures 3 and 4 ) or the invading front penetrated a certain distance into the uterine tissue before stopping (see Figures 5 and 6).

Figures 3-6 illustrate the way in which changes in the rate of protease production $\left(k_{2}\right)$ and the rate of inhibitor production $\left(k_{4}\right)$ affect trophoblast implantation. For moderale values of $k_{2}$ invasion 

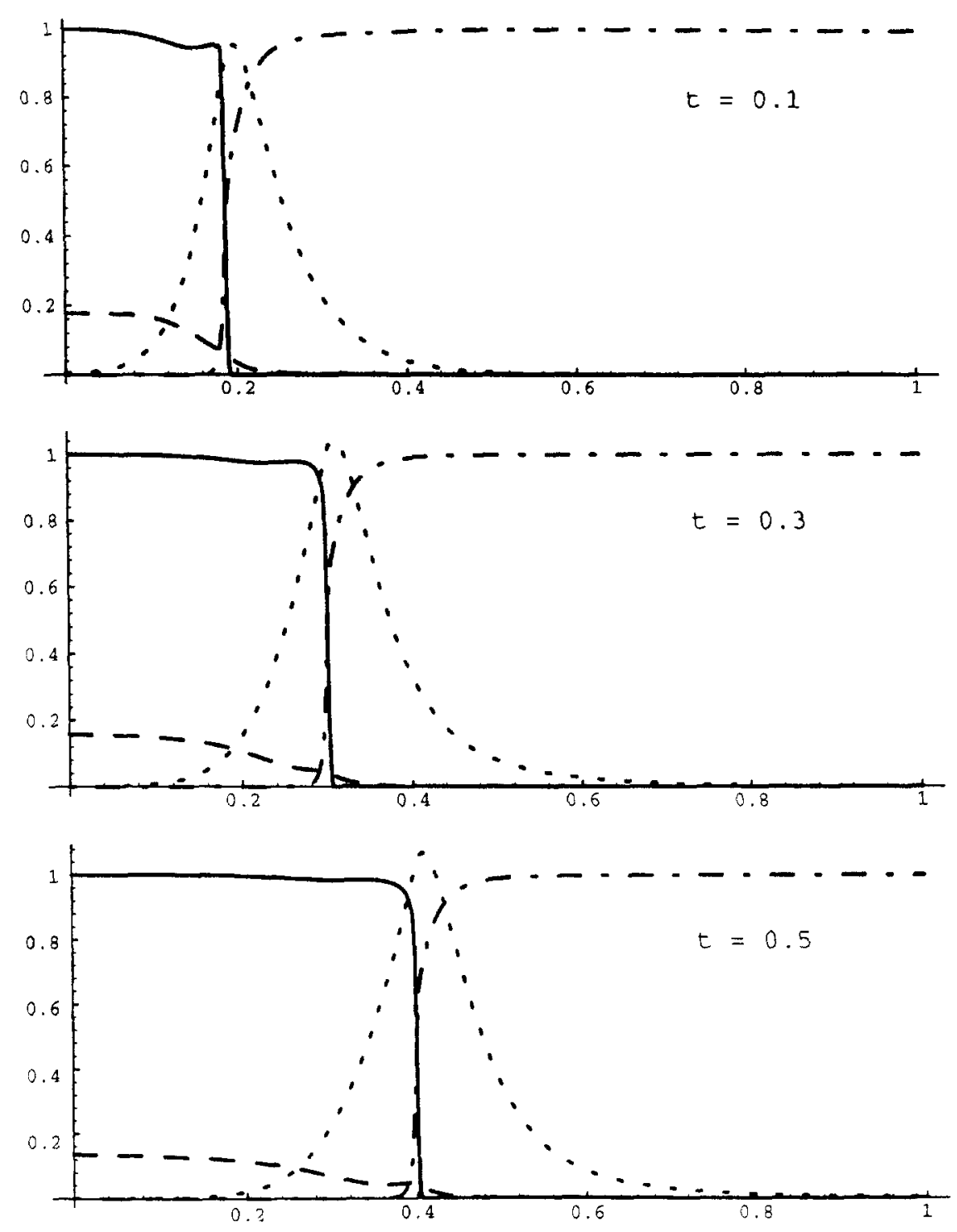

FIGURE 4 An illustration of the affect on invasion of an increase in the rate of inhibitor production ( $k_{4}$ increased from 10 to 50 ). Key: as per Figure 3. Parameter values: as per Figure 3 except that $k_{4}=50$.

is successful and a travelling wave of invading trophoblast cells is established (see Figure 1). Protease $(u)$ produced by cells at the leading edge of the invading trophoblast front $(n)$ degrades the uterine tissue $(r)$ directly ahead of the trophoblast front. As it is degraded, the tissue releases inhibitor (v) which subsequently binds with the protease, thereby establishing a spatial gradient in $v$. The trophoblast cells chemotactically migrate into the region of degraded tissue in response to the gradient in $v$. Once there, they proliferate and produce more protease allowing further uterine tissue degradation and trophoblast invasion to occur.

The simulations presented in Figure 2 illustrate the effect of increasing the rate of inhibitor production $\left(k_{4}\right)$. Notably, there is a reduction in the extent of the region of degraded uterine tissue ahead of the trophoblast front. Also in this case the peak in the trophoblast density at the invading front is reminiscent of the brush border effect observed in angiogenesis 

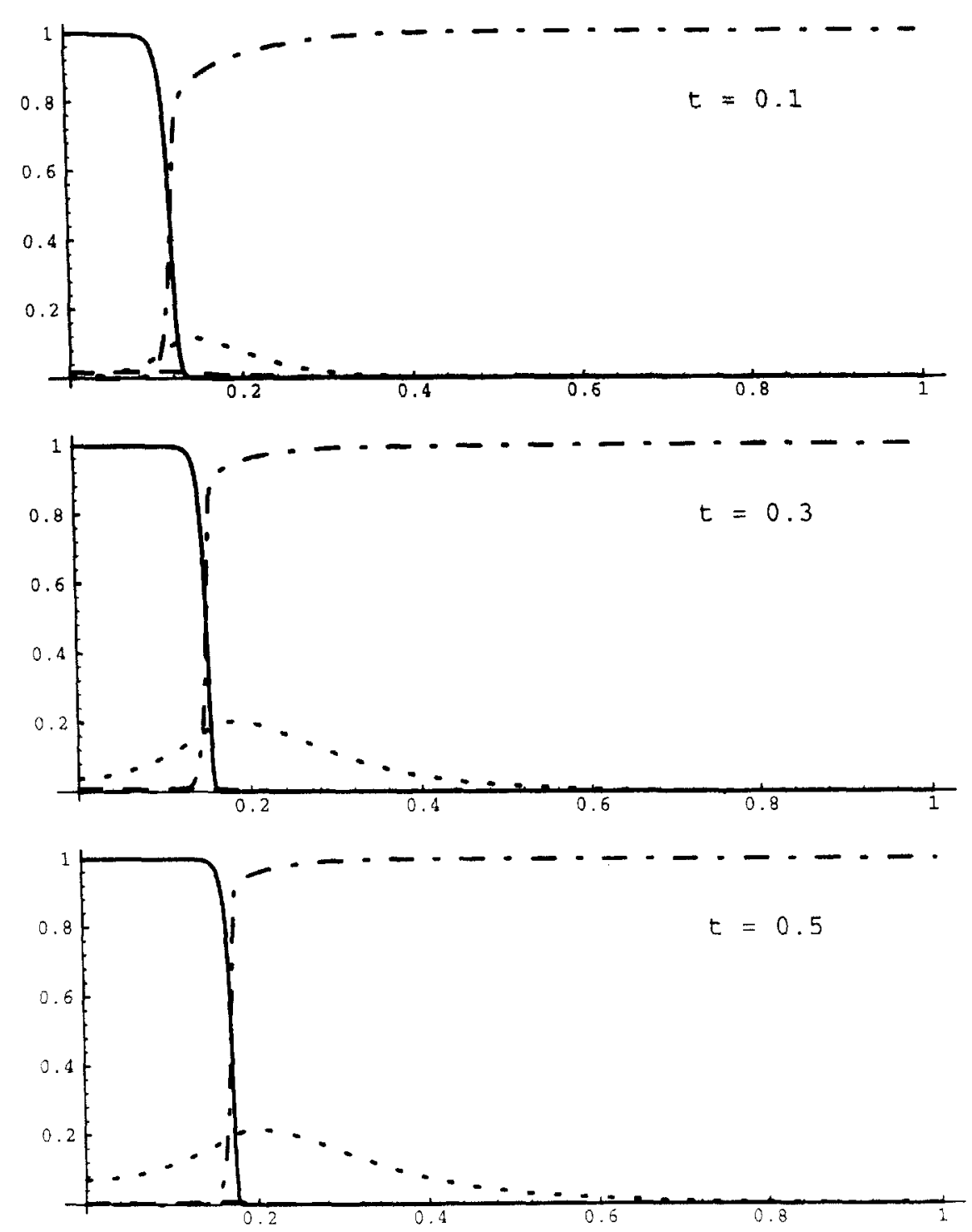

FIGURE 5 An illustration of failed invasion brought about by insufficient protease production ( $k_{2}$ decreased from 10.0 to 0.5 ). Key: as per Figure 3. Parameter values: as per Figure 3 except that $k_{2}=0.5$.

(Pettet et al., 1996b). It is a consequence of the trophoblast cells' chemotactic response to the gradient of inhibitor at the invading front.

For small values of $k_{2}$ (see Figure 3 ) invasion is unsuccessful because there is insufficient protease production. This prevents adequate degradation of the uterine tissue ahead of the trophoblasts. The absence of a degraded region of uterine tissue ahead of the invading front prevents the trophoblasts from either migrating up the inhibitor gradient via chemotaxis or from proliferating, the reduced proliferation being a response to local overcrowding.

For large values of $k_{2}$ (see Figure 4) invasion is also unsuccessful. In this case invasion fails because the region of degraded uterine tissue becomes too large. Consequently the inhibitor is concentrated so far ahead of the trophoblasts that the inhibitor gradient in the vicinity of the trophoblast front is negligible and further chemotactic motion of the trophoblasts is halted. 

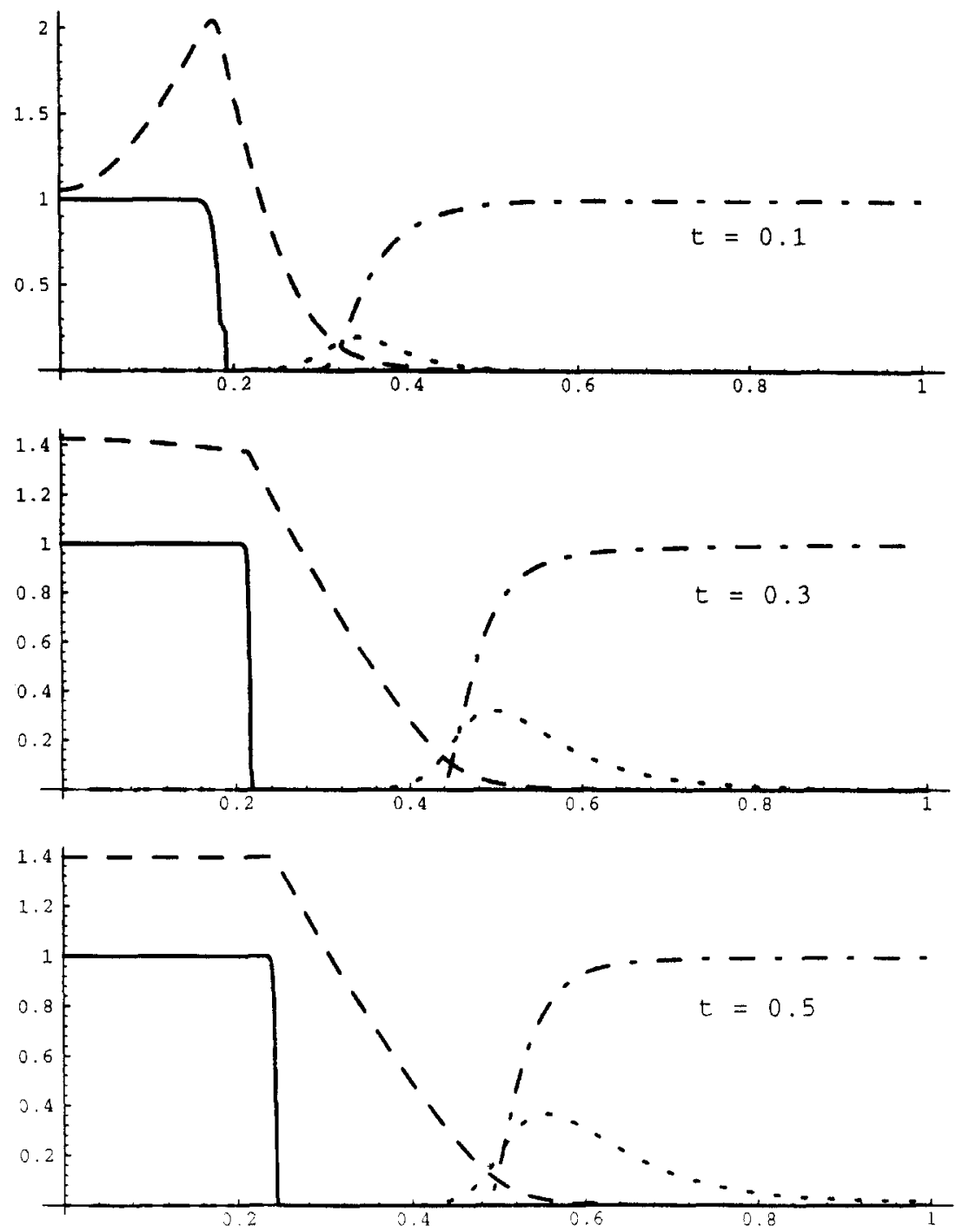

FIGURE 6 An illustration of failed invasion brought about by excessive protease production ( $k_{2}$ increased from 10 to 50). Key: as per Figure 3. Parameter values: as per Figure 3 except that $k_{2}=50$.

The numerical results presented above suggest that both protease expression and chemotaxis are key features of early placental development. To test the latter assertion we performed further numerical experiments to investigate the effect of neglecting the chemotactic response of the trophoblasts to spatial gradients in the inhibitor. For a wide range of parameter values, with $\chi=0$ in equation (1), our simulations failed to establish a travelling wave. By contrast, with $\chi>0$ the simulations evolved to travelling waves for a wide range of parameter values. Given that in the present context the desired behaviour corresponds to a steady travelling wave, we postulate that a chemotactic response to a gradient in a maternally-derived chemical plays an important role in trophoblast invasion.

\section{CONCLUSIONS}

It is clear that the process of placental development is complex, involving many inter-related mechanisms. In this paper we have presented a 
mathematical model that describes the initial stages of placental development, during which the trophoblast cells that comprise the anchoring and floating villi of the developing embryo invade the uterine tissue. In so doing we have not focused on specific ECM degrading enzymes or their inhibitors, but rather have considered a generic trophoblastderived protease and a generic uterine tissue-derived inhibitor. We remark that our model of protease/ inhibitor regulated trophoblast invasion is sufficiently general in form to be applicable to other invasion processes such as cancer invasion (Byrne and Chaplain, 1996; Perumpanani et al., 1997; StetlerStevenson et al., 1993; Tsuboi and Rifkin, 1990) and angiogenesis (Bennet and Schultz, 1993; Pettet et al., 1996a, b).

By restricting our mathematical model to a description of the initial stages of placental development, any consideration of the mechanisms which in normal cases may halt trophoblast invasion have been neglected. Consequently our model does not distinguish between normal trophoblast invasion and choriocarcinoma: in both cases the trophoblasts successfully penetrate the uterine tissue. Thus the establishment of an invading front of trophoblast cells may be interpreted as either unregulated invasion (a characteristic of choriocarcinoma) or as successful trophoblast implantation prior to the involvement of mechanisms, not included in the present model, that are responsible for halting the invasion process. In vivo, an increase in the level of growth factors, such as TGF- $\beta$, has been suggested as a mechanism for halting invasion in normal implantation. These chemicals are believed to down-regulate protease production, upregulate inhibitor production and cause the trophoblasts to mature into large, immobile cytotrophoblasts (Graham, 1997). Such observations have led to speculation that choriocarcinomas may result when this regulatory mechanism fails (Graham et al., 1994). By extending our model to explicitly include growth factors such as TGF- $\beta$ we hope to distinguish between normal and pathological implantation.

Even though this model is unable to distinguish between normal implantation and choriocarcinoma, the numerical results presented in Section 3 show that it can distinguish between failed trophoblast implantation and normal implantation; normal implantation is represented by a wave-like profile of trophoblast cells which travels with constant speed through the uterine tissue whereas halted implantation corresponds to the failure of such a profile to be established.

By manipulating the model parameters we were able to generate a number of hypotheses that could be tested experimentally. For example, by varying the rate of protease production (see Figures 3, 5 and 6) we observe two mechanisms that may be associated with failed trophoblast implantation. Specifically, if the rate of protease production is either too high or too low then invasion halts prematurely. In the case of elevated protease production (see Figure 6) invasion fails as the elevated protease production causes excessive uterine tissue degradation, thereby reducing the potential for the degraded region to produce inhibitor. This, in turn, results in the establishment of a region of the trophoblasts cells in which the inhibitor gradient vanishes, precluding any further chemotactic migration of the trophoblasts. Such behaviour is known to halt the spread of solid tumours grown in vitro (StetlerStevenson et al., 1993). It should be possible to test whether excess protease production could lead to premature arrest of placental development by studying the outgrowth of mouse blastocysts cultured in media treated with different concentrations of a particular protease (Suenaga et al., 1997). This assay could also be used to test whether insufficient protease levels can cause trophoblast invasion to fail (see Figure 5) (Landman and Pettet, 1997). In this case we hypothesise that invasion ceases because there is insufficient degradation of the uterine tissue to accommodate further trophoblast cell proliferation and migration.

By varying the chemotactic coefficient ( $\chi$ in equation (1)) we observed that, in the absence of chemotaxis, trophoblast invasion failed whereas in the presence of chemotaxis successful implantation occurred for a wide range of parameter values. These results lead us to postulate that chemotactic 
migration is a key feature of trophoblast locomotion. Whilst chemicals with the potential for eliciting chemotactic responses in other cells are known to be present within the uterine tissue, to our knowledge there is at present no experimental evidence supporting or refuting our claim. Recent experiments demonstrating that trophoblast cells are sensitive to thermal gradients and will migrate towards sources of heat (thermotaxis) provide supporting evidence for the ability of trophoblast cells to migrate in response to chemical gradients (Higazi et al., 1996). Our hypothesis could be tested by using the in vitro assay of Suenaga et al. (1997) or by using a Boyden chamber (Boyden, 1962). The Boyden chamber enables experimentalists to assess whether particular chemicals influence cellular migration. By performing repeated experiments in which different chemical gradients are established within the Boyden chamber it should be possible to determine whether a particular chemical elicits a chemotactic response from trophoblast cells.

To summarise, in this paper we have presented a simple model of early placental development as characterised by the invasion of trophoblast cells into maternal uterine tissue. There are many ways in which our model could be extended. For example, we could study the effect that nutrients such as oxygen exert on trophoblast proliferation and differentiation. Equally, the inclusion of growth factors such as TGF- $\beta$ may enable us to distinguish between normal placental development and choriocarcinoma. By performing a series of numerical experiments we have generated a number of hypotheses which could be tested experimentally. In particular, we suggest that both chemotactic migration of trophoblast cells and an appropriate rate of protease production (neither too high nor too low) are necessary for normal placental development.

\section{Acknowledgments}

HMB, MAJC and GJP thank Queensland University of Technology and CiSSaIM for financial support, without which this work would not have been possible. They are also grateful to $M$. Harvey and J. Aplin for valuable discussions.

\section{References}

Anderson, A. R. A. and Chaplain, M. A. J. (1998) Continuous and discrete mathematical models of tumour-induced angiogenesis. Bull. Math. Biol., 60, (in press).

Bennet, N. T. and Schultz, G. S. (1993) Growth factors and wound healing: part I role in normal and chronic wound healing. Am. J. Surg. 166, 74-81.

Bischof, P. and Campana, A. (1996) A model for implantation of the human blastocyst and early placentation. Human Reprod. Update, 2, 262-270.

Boyden. S. V. (1962) The chemotactic effect of mixtures of antibody and antigen on polymorphonuclear leukocytes. $J$. Exp. Med., 115, 453-466.

Bray, D. (1992) Cell Movements Garland Publishing: New York. Burrows, T. D., King, A. and Loke, Y. W. (1996) Trophoblast migration during human placental implantation. Human Reprod. Update, 2, 307-321.

Byrne, H. M. and Chaplain, M. A. J. (1996) Modelling the role of cell-cell adhesion in the growth and development of carcinomas. Math. Comput. Modelling, 24, 1-17.

Carlson, B. M. (1994) Human embryology and developmental biology. Missouri: Mosby-Year Book. Inc.

Chaplain, M. A. J. and Stuart, A. M. (1993) A model mechanism for the chemotactic response of endothelial cells to tumour angiogenesis factor. IMA J. Math. Appl. Med. Biol., 10, $149-168$.

Elliott, C. M. and Ockendon, J. R. (1982) Weak and variational methods for moving boundary problems, Pitman, London.

Endos, A. C. (1991) Implantation. In Encyclopaedia of human biology, 4, New York: Academic.

Genbacev, O., Joslin, R, Damsky, C. H., Polliotti, B. M. and Fisher. S. J. (1996) Hypoxia alters early gestation human cytotrophoblast differentiation in-vitro and modcls the placental defects that occur in preeclampsia. J. Clin. Invest., 97. $540-550$.

Genbacev, O., Zhou. Y., Ludlow, J. and Fisher, S. (1997) Regulation of human placental development by oxygen tension. Science, 277. $1669-1672$.

Giannelli, G.. FalkMarzillicr, J., Schiraldi, O., Stetler-Stevenson. W. G. and Quaranta. V. (1997) Induction of cell migration by matrix metalloprotease-2 cleavage of laminin-5. Science, 277 , $225-228$.

Graham, C. H. (1997) Effect of transforming growth factor- $\beta$ on the plasminogen activator system in cultured first trimester human cytotrophoblasts. Placenta, 18, 137-143.

Graham, C. H., Connelly, I., MacDougall, J. R., Kerbel, R. S., Stetler-Stevenson, W. G. and Lala, P. K. (1994) Resistance of malignant trophoblast cells to both the antiproliferative and anti-invasive effects of transforming growth-factor- $\beta$. Exp. Cell Res., 214, $93-99$.

Graham, C. H. and Lala, P. K. (1992) Mechanisms of placental invasion of the uterus and their control. Biochem. Cell Biol., 70, $867-874$.

Harvey, M. B., Leco. K. J., Arcellana-Panlilio, M. Y., Lhang. X., Edwards, D. R, and Schult7, G. A. (1995) Proteinase expression in early mouse embryos is rcgulated by leukaemia inhibitory factor and epidermal growth factor. Development, 121, $1005-1014$.

Higazi, A. A., Kniss, D., Manuppello, J., Barnathan, E. S. and Cincs, D. B. (1996) Thermotaxis of human trophoblastic cells. Placenta, 17(8), 683-687.

Klciner, D. E. and Stctler-Stevenson, W. G. (1993) Structural biochemistry and activation of matrix metallo-proteases. Curr. Opin. Cell Biol. . 5, 891-897. 
Landman, K. A. and Pettet, G. J. (1997) Modelling the action of proteinase and inhibitor in tissue invasion. Mathematical Biosciences (submitted).

Perumpanani, A. J., Sherratt, J. A., Norbury. J. and Byrne, H. M. (1997) Biological inferences from a mathematical model for malignant invasion. Invasion and Metastasis, 16, 209-221.

Pettet, G. J., Byrne, H. M., McElwain, D. L. S and Norbury, J (1996a) A model of wound-healing angiogenesis in soft tissue. Math. Biosci., 136, 35-63.

Pettet, G., Chaplain, M. A. J., McElwain, D. L. S. and Byrne, H. M. (1996b) On the role of angiogenesis in wound healing. Prac. Roy. Soc. Lond. B, 263, 1487-1493.

Redman, C. W. (1997) Cytutrophoblasts: masters of disguise Nature Medicine, 3, 610-611.
Stetler-Stevenson, W. G.. Aznavoorian, S. and Liotta, L. A (1993) Tumour cell interactions with the extra-cellular matrix during invasion and metastasis. Ann. Rev. Cell. Biol., 9 $541-573$.

Strickland. S. and Richards, W. G. (1992) Invasion of the trophoblasts. Cell, 71, 355-357.

Suenaga, A., Tachi, C., Tojo, H., Tanaka, S. and Taketani, Y. (1996) Quantitative analysis of the spreading of the mouse trophoblast in-vitro - a model for early invasion. Placenta. $17,583-590$ ).

Tsuboi, R. and Rifkin, D. B. (1990) Bimodal relationship between invasion of the amniotic membrane and plasminogen activator activity. Int. J. Cancer, 46, 56-60. 


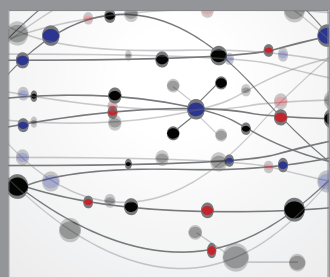

The Scientific World Journal
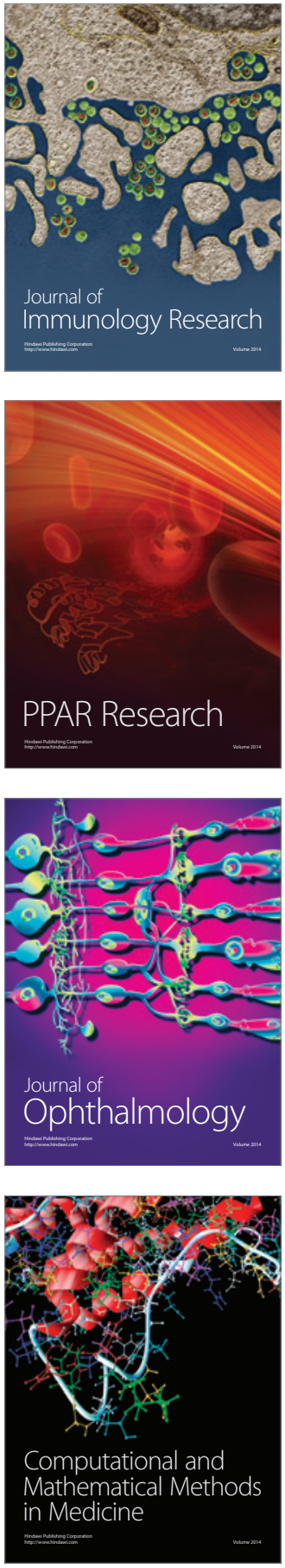

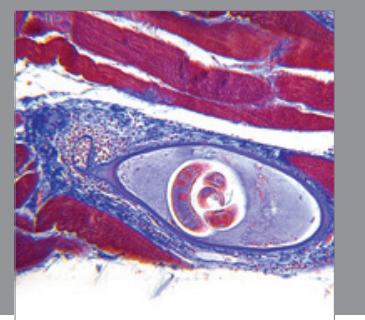

Gastroenterology

Research and Practice
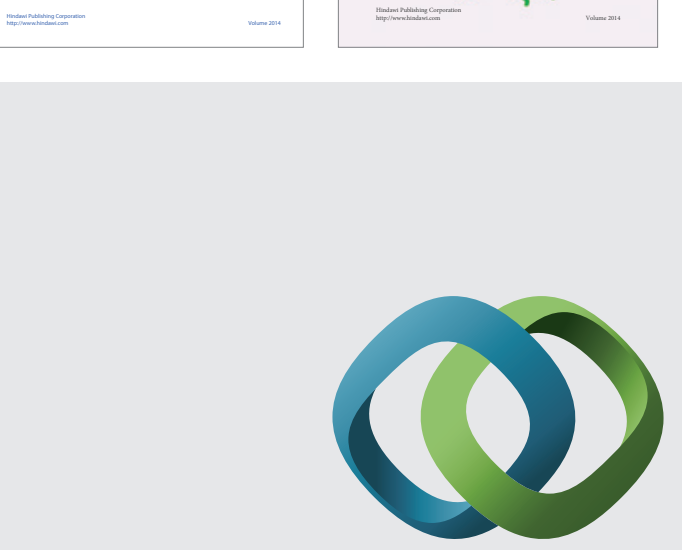

\section{Hindawi}

Submit your manuscripts at

http://www.hindawi.com
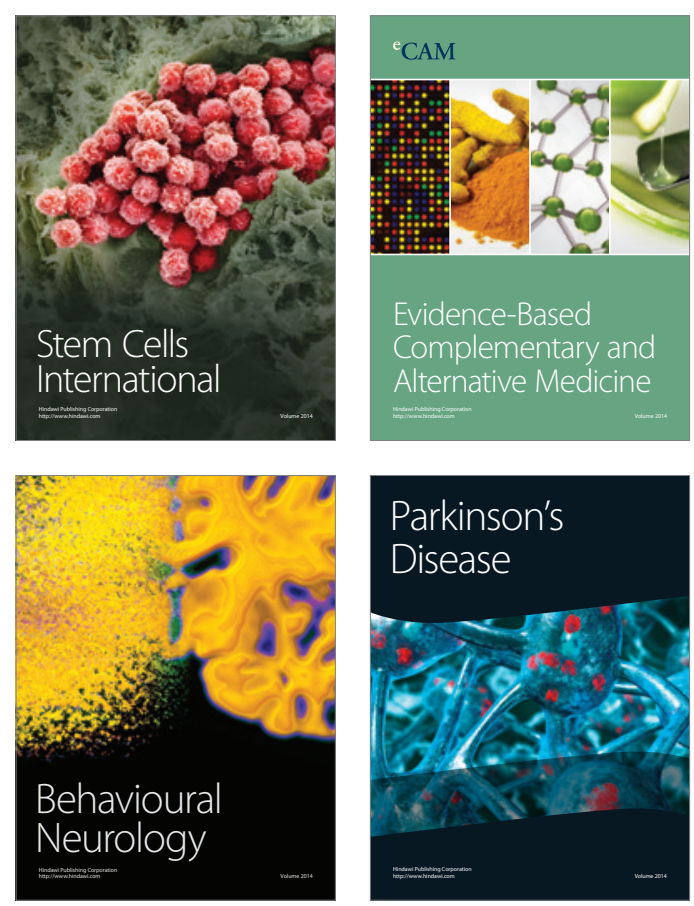

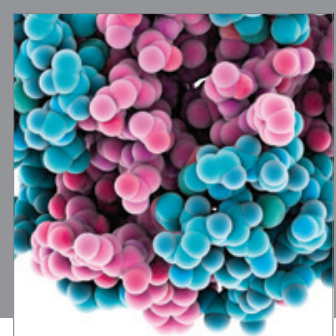

Journal of
Diabetes Research

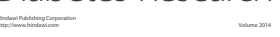

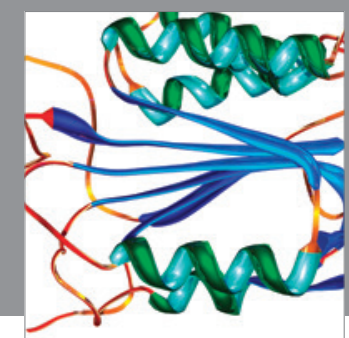

Disease Markers
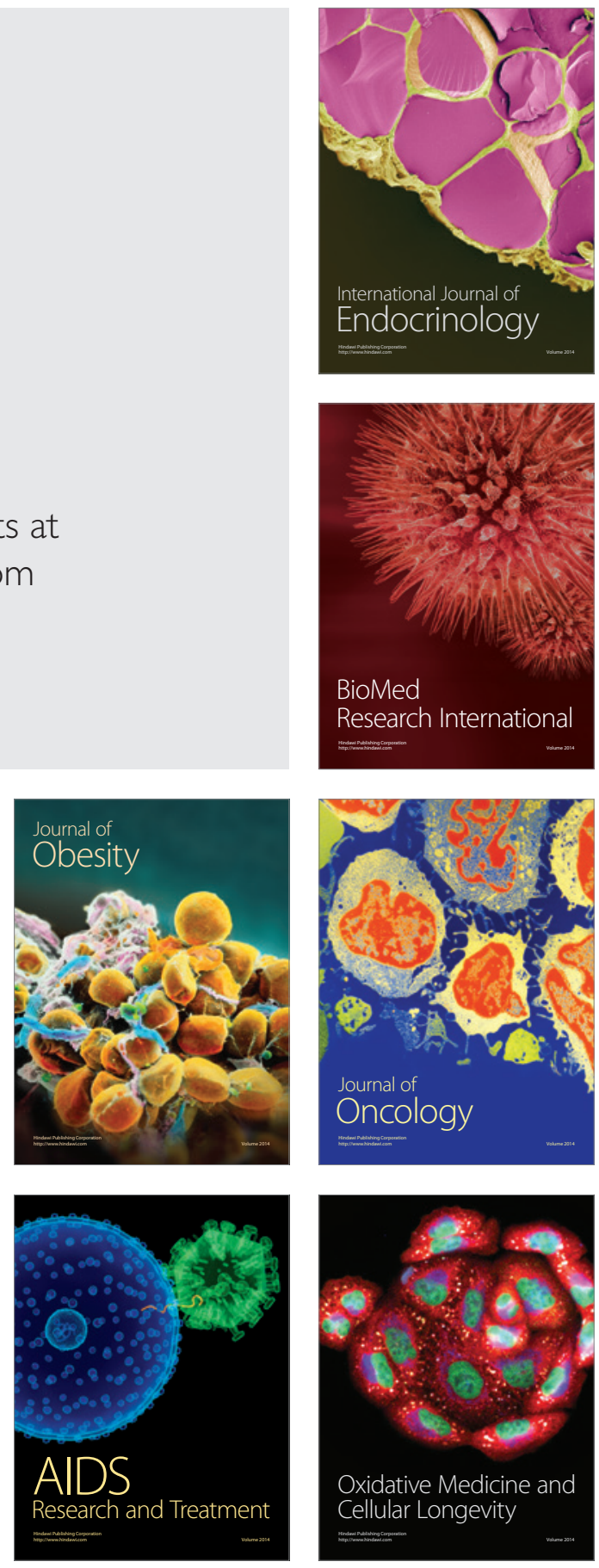